\title{
Stochastic maximum principle for delayed backward doubly stochastic control systems
}

\author{
Jie Xua,b, Yuecai Han ${ }^{a, *}$ \\ a School of Mathematics, Jilin University, Changchun, 130012, China. \\ ${ }^{b}$ College of Sciences, Jilin Institute of Chemical Technology, Jilin, 132022, China. \\ Communicated by M. Eslamian
}

\begin{abstract}
In this paper, we investigate a class of doubly stochastic optimal control problems that the state trajectory is described by backward doubly stochastic differential equations with time delay. By means of martingale representation theorem and contraction mapping principle, the existence and uniqueness of solution for the delayed backward doubly stochastic differential equation can be guaranteed. When the control domain is convex, we deduce a stochastic maximum principle as a necessary condition of the optimal control by using classical variational technique. At the same time, under certain assumptions, a sufficient condition of optimality is obtained by using the duality method. In the last section, we give the explicit form of the optimal control for delayed doubly stochastic linear quadratic optimal control problem by our stochastic maximal principle. (C)2017 all rights reserved.
\end{abstract}

Keywords: Stochastic maximum principle, doubly stochastic differential equation, time delay, optimal control. 2010 MSC: 60H10, 93E20.

\section{Introduction}

Pardoux and Peng [11] first introduced the backward doubly stochastic differential equations (BDSDEs for short) to introduce a probabilistic presentation of certain quasilinear stochastic partial differential equations (SPDEs for short). Since then, the theory of BDSDEs has been developed rapidly by many researchers. Han et al. [6] investigated the maximum principle for backward doubly stochastic control systems. Shi et al. [15] gave a comparison theorem for BDSDEs. Lin [8] obtained a generalized existence theorem for BDSDEs. Matoussi-Scheutzow [9] studied BDSDEs and introduced some applications in SPDEs.

On the other hand, recently, Peng and Yang [13] introduced a new type of backward stochastic differential equations (BSDEs for short) that called anticipated BSDEs. Xu [17] and Zhang [19] considered the similar problem for the doubly stochastic differential equations (DSDEs for short). Zhang [19] introduced one kind of anticipated BDSDEs and present several comparison theorems in one dimensional case. $\mathrm{Xu}$ [17] studied anticipated BDSDEs and obtained a comparison theorem for the solutions of these equations.

\footnotetext{
*Corresponding author

Email addresses: aqie990132@126.com (Jie Xu), hanyc@jlu.edu.cn (Yuecai Han)
} 
Sometimes we find many stochastic problems have the relation not only with the present time but also with their past history, which can be identified as time delay problems $[1,2,7,16]$. Based on the abundant literature, we investigate a class of doubly stochastic optimal control problems which the state equation of the control system is described by delayed BDSDEs. Stochastic maximum principle as a necessary condition of the optimal control is a principle approach in solving optimization problems. Stochastic maximum principle has important applications in many fields. Yong [18] gave the optimality variational principle for controlled forward backward stochastic differential equations with mixed initial-terminal conditions. Hafayed et al. [3-5] gave the maximum principle and application to finance in mean field optimal control for forward backward stochastic systems and so on.

We know that one of the classical approaches in solving optimization problems is to derive a set of necessary conditions that the optimal control should satisfy. We will utilize the duality relation between the state equation and its adjoint equation to obtain the maximum principle for the delayed doubly stochastic control problems as a necessary and sufficient condition of the optimal control.

In this paper, we discuss only one variable contained time delay in the state equations. Of course, there are some other cases. For example, two or more variables contain time delay in the same time. The existence and uniqueness conditions of the solution of the state equation will be strengthened when several variables contain time delay. And the existence and uniqueness of the solution of the adjoint equation will be restricted at the same time. We all know that it is not suitable for practical applications when there is too many restrictions. We will continue to study this research, and extend the current work to more general cases.

The rest of this paper is organized as follows. In Section 2, we state the delayed doubly stochastic optimal control problem and some necessary notations. In Section 3, the existence and uniqueness of solution for delayed BDSDEs is proved in certain space. Then we deduce a stochastic maximum principle as a necessary condition of the optimal control should satisfy, and prove a sufficient condition of optimality under certain assumptions. In Section 4, we discuss the delayed doubly stochastic Linear quadratic (LQ) problem to illustrate our theoretical results.

\section{Preliminaries}

Let $(\Omega, \mathcal{F}, P)$ be a probability space. Let $\{\mathrm{W}(\mathrm{t}): 0 \leqslant \mathrm{t} \leqslant \mathrm{T}\}$ and $\{\mathrm{B}(\mathrm{t}): 0 \leqslant \mathrm{t} \leqslant \mathrm{T}\}$ be two mutually independent standard Brownian motions defined on $(\Omega, \mathcal{F}, P)$, with values respectively in $R^{m}$ and in $\mathrm{R}^{\mathrm{d}}$. Let $\mathcal{N}$ denote the class of P-null sets of $\mathcal{F}$. For each $t \in[0, T]$, we define $\mathcal{F}_{t}:=\mathcal{F}_{t}^{w} \vee \mathcal{F}_{t, T}^{B}$, where $\mathcal{F}_{t}^{w}=\mathcal{N} \vee \sigma\{W(r)-W(0): 0 \leqslant r \leqslant t\}, \mathcal{F}_{t, T}^{B}=\mathcal{N} \vee \sigma\{B(r)-B(t): t \leqslant r \leqslant T\}$. Let $M^{2}\left(0, T ; R^{n}\right)$ denote the set of all classes of $\left(d t \times d P\right.$ a.e. ) $\mathcal{F}_{t}$ measurable stochastic process $\varphi(t)$ satisfying $E \int_{0}^{T}|\varphi(t)|^{2} d t<+\infty$. Similarly, $S^{2}\left(0, T ; R^{n}\right)$ denotes the set of continuous $n$-dimensional $\mathcal{F}_{t}$ measurable stochastic process $\varphi(t)$ satisfying $\operatorname{Eup}_{\mathrm{t} \in[0, \mathrm{~T}]}|\varphi(\mathrm{t})|^{2}<+\infty$.

For a given $\varphi(t), \psi(t) \in M^{2}\left(0, T ; R^{n}\right)$, one can define the forward Itô's integral $\int_{0}^{0} \varphi(s) d \overrightarrow{W(s)}$ and the backward Itô's integral $\int_{0}^{T} \psi(s) d \overleftarrow{B(s)}$. They are both in $M^{2}\left(0, T ; R^{n}\right)$ (see $[10,14]$ for details). Moreover,

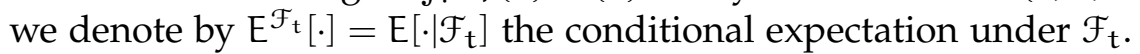

For a convex subset $\mathrm{V} \subset \mathrm{R}^{\mathrm{k}}$, we define the set of $\mathrm{U}[0, \mathrm{~T}]$ of admissible controls to be the class of measurable processes satisfying the following conditions:

(i) $\mathfrak{u}(\cdot)$ is $\mathcal{F}_{t}$ adapted, $u(t) \in V, t \in[0, T]$;

(ii) $\mathrm{E} \int_{0}^{\mathrm{T}}|\mathrm{u}(\mathrm{t})|^{2} \mathrm{dt}<+\infty$.

Let $y_{\delta}(t)=y(t-\delta)$. We consider the following delayed doubly stochastic differential equation

$$
\left\{\begin{aligned}
d y(t) & =f\left(t, y(t), y_{\delta}(t), z(t), u(t)\right) d t+g\left(t, y(t), y_{\delta}(t), z(t), u(t)\right) d \overrightarrow{W(t)}-z(t) d \overleftarrow{B(t)}, t \in[0, T] \\
y(t) & =\varphi(t), t \in[-\delta, 0]
\end{aligned}\right.
$$


with the cost functional

$$
J(u(\cdot))=E\left\{\int_{0}^{T} l(t, y(t), z(t), u(t)) d t+\Phi(y(T))\right\}
$$

Our optimal control problem can be stated as maximizing the cost functional over $\mathrm{U}[0, \mathrm{~T}]$. For optimal control $u^{*}(\cdot)$ satisfying

$$
\mathrm{J}\left(\mathrm{u}^{*}(\cdot)\right)=\sup _{\mathfrak{u}(\cdot) \in \mathrm{U}[0, \mathrm{~T}]} \mathrm{J}(\mathfrak{u}(\cdot)),
$$

the corresponding $\left(\mathrm{y}^{*}(\cdot), \mathrm{z}^{*}(\cdot), \mathrm{u}^{*}(\cdot)\right)$ is called an optimal triple.

We assume that the following conditions hold:

(H1) $f: \Omega \times[0, T] \times R^{n} \times R^{n} \times R^{n \times m} \times R^{k} \rightarrow R^{n}, g: \Omega \times[0, T] \times R^{n} \times R^{n} \times R^{n \times m} \times R^{k} \rightarrow R^{n \times d}$. $f$ and $g$ are continuously differentiable with respect to $y, y_{\delta}, z, u$, and their derivatives are bounded.

(H2) There exists a constant $C>0$, such that for any $(\omega, t) \in \Omega \times[0, T], y, y^{\prime} \in R^{n}, z, z^{\prime} \in R^{n \times m}$, $\xi, \xi^{\prime} \in \mathrm{R}^{\mathrm{n}}$, the function $\mathrm{f}$ satisfies

$$
\left|f(t, y, z, \xi)-f\left(t, y^{\prime}, z^{\prime}, \xi^{\prime}\right)\right|^{2} \leqslant C\left(\left|y-y^{\prime}\right|^{2}+\left|z-z^{\prime}\right|^{2}+\left|\xi-\xi^{\prime}\right|^{2}\right) .
$$

(H3) There exists a constant $L>0$, such that for all $t \in[0, T]$ and for all nonnegative and integrable function $\theta(\cdot), \int_{t}^{T} \theta(s-\delta) d s \leqslant \int_{t}^{T} L \theta(s) d s$.

(H4) There exist constants $C>0$ and $0<\alpha<\frac{\mathrm{L}}{1+\mathrm{L}}$, such that for any $(\omega, \mathrm{t}) \in \Omega \times[0, \mathrm{~T}], \mathrm{y}, \mathrm{y}^{\prime} \in \mathrm{R}^{\mathrm{n}}, z, z^{\prime} \in$ $\mathrm{R}^{\mathrm{n} \times \mathrm{m}}, \xi, \xi^{\prime} \in \mathrm{R}^{\mathrm{n}}$, the function $\mathrm{g}$ satisfies

$$
\left|g(t, y, z, \xi)-g\left(t, y^{\prime}, z^{\prime}, \xi^{\prime}\right)\right|^{2} \leqslant C\left(\left|y-y^{\prime}\right|^{2}+\left|\xi-\xi^{\prime}\right|^{2}\right)+\alpha\left|z-z^{\prime}\right|^{2} .
$$

We need the following results that can be seen in Pardoux and Peng [11].

Lemma 2.1 ([11]). Let $\alpha \in S^{2}\left(0, T ; R^{n}\right), \beta \in M^{2}\left(0, T ; R^{n}\right), \gamma \in M^{2}\left(0, T ; R^{n} \times R^{m}\right)$, and $\delta \in M^{2}\left(0, T ; R^{n} \times R^{m}\right)$ be such that

$$
\alpha(t)=\alpha(0)+\int_{0}^{t} \beta(s) d s+\int_{0}^{t} \gamma(s) d \overleftarrow{B(s)}+\int_{0}^{t} \delta(s) d \overrightarrow{W(s)}, t \in[0, T]
$$

Then

$$
\begin{aligned}
|\alpha(\mathrm{t})|^{2}= & |\alpha(0)|^{2}+2 \int_{0}^{\mathrm{t}}(\alpha(\mathrm{s}), \beta(\mathrm{s})) \mathrm{d} s+2 \int_{0}^{\mathrm{t}}(\alpha(\mathrm{s}), \gamma(\mathrm{s}) \mathrm{d} \overleftarrow{\mathrm{B}(\mathrm{s})})+2 \int_{0}^{\mathrm{t}}(\alpha(\mathrm{s}), \delta(\mathrm{s}) \mathrm{d} \overrightarrow{\mathrm{W}(\mathrm{s})}) \\
& -\int_{0}^{\mathrm{t}}\|\gamma(\mathrm{s})\|^{2} \mathrm{~d} s+\int_{0}^{\mathrm{t}}\|\delta(\mathrm{s})\|^{2} \mathrm{~d} s, \\
\mathrm{E}|\alpha(\mathrm{t})|^{2}= & \mathrm{E}|\alpha(0)|^{2}+2 \mathrm{E} \int_{0}^{\mathrm{t}}(\alpha(\mathrm{s}), \beta(\mathrm{s})) \mathrm{d} s-\mathrm{E} \int_{0}^{\mathrm{t}}\|\gamma(\mathrm{s})\|^{2} \mathrm{~d} s+\mathrm{E} \int_{0}^{\mathrm{t}}\|\delta(\mathrm{s})\|^{2} \mathrm{~d} s .
\end{aligned}
$$

More generally, if $\phi \in \mathrm{C}^{2}\left(\mathrm{R}^{\mathrm{n}}\right)$, then

$$
\begin{aligned}
\phi(\alpha(\mathrm{t}))= & \phi(\alpha(0))+\int_{0}^{\mathrm{t}}\left(\phi^{\prime}(\alpha(s)), \beta(\mathrm{s})\right) \mathrm{d} s+2 \int_{0}^{\mathrm{t}}\left(\phi^{\prime}(\alpha(s)), \gamma(\mathrm{s}) \mathrm{d} \overleftarrow{\mathrm{B}(\mathrm{s})}\right)+\int_{0}^{\mathrm{t}}\left(\phi^{\prime}(\alpha(s)), \delta(\mathrm{s}) \mathrm{d} \overrightarrow{\mathrm{W}(\mathrm{s})}\right) \\
& -\frac{1}{2} \int_{0}^{\mathrm{t}} \operatorname{Tr}\left[\phi^{\prime \prime}(\alpha(\mathrm{s})) \gamma(\mathrm{s}) \gamma(\mathrm{s})^{\top}\right] \mathrm{d} s+\frac{1}{2} \int_{0}^{\mathrm{t}} \operatorname{Tr}\left[\phi^{\prime \prime}(\alpha(\mathrm{s})) \delta(\mathrm{s}) \delta(\mathrm{s})^{\top}\right] \mathrm{d} s .
\end{aligned}
$$

\section{Main results}

Before we deduce the maximum principle, we need the following conclusion. Given a $u(\cdot) \in U[0, T]$, there exists a unique pair $(y(\cdot), z(\cdot))$ solves equation (2.1), that is the following theorem. 
Theorem 3.1. Assume that $\mathrm{f}$ and $\mathrm{g}$ satisfy (H1)-(H4). Then there exists a unique solution satisfying the following delayed doubly stochastic differential equation

$$
\left\{\begin{aligned}
d y(t) & =f\left(t, y(t), y_{\delta}(t), z(t)\right) d t+g\left(t, y(t), y_{\delta}(t), z(t)\right) d \overrightarrow{W(t)}-z(t) d \overleftarrow{B(t)}, t \in[0, T] \\
y(t) & =\varphi(t), \quad t \in[-\delta, 0] .
\end{aligned}\right.
$$

Proof. To prove Theorem 3.1, we first consider the simple BDSDEs

$$
d y(t)=f(t) d t+g(t) d \overrightarrow{W(t)}-z(t) d \overleftarrow{B(t)}, t \in[0, T]
$$

Similar to the proof in the [11] and [12], by using the Itô's martingale representation theorem and contraction mapping principle, there exist a unique pair $(y, z)$ which solves equation (3.2).

Let us define a space $\mathcal{B}$ and introduce a norm in it:

$$
\|(y(\cdot), z(\cdot))\|_{\beta}=\left\{E \int_{0}^{T} e^{-\beta t}\left(\frac{\beta}{2}|y(t)|^{2}+|z(t)|^{2}\right)\right\}^{\frac{1}{2}}, \beta>0 .
$$

Let

$$
\left\{\begin{aligned}
d y(t) & =f\left(t, Y(t), Y_{\delta}(t), Z(t)\right) d t+g\left(t, Y(t), Y_{\delta}(t), Z(t)\right) d \overrightarrow{W(t)}-z(t) d \overleftarrow{B(t)}, \quad t \in[0, T] \\
y(t) & =\varphi(t), t \in[-\delta, 0] .
\end{aligned}\right.
$$

For each $(Y(t), Z(t)) \in \mathcal{B}$, there exists a unique solution $(y(t), z(t))$ satisfying the above equation. So we define a mapping $h: \mathcal{B} \rightarrow \mathcal{B}$ such that $h(Y(\cdot), Z(\cdot))=(y(\cdot), z(\cdot))$. We want to prove that $h$ is a contraction mapping under the norm $\|\cdot\|_{\beta}$.

For two arbitrary elements $\left(Y^{1}(\cdot), Z^{1}(\cdot)\right),\left(Y^{2}(\cdot), Z^{2}(\cdot)\right) \in \mathcal{B}$, let

$$
\left(y^{1}(\cdot), z^{1}(\cdot)\right)=h\left(Y^{1}(\cdot), Z^{1}(\cdot)\right),\left(y^{2}(\cdot), z^{2}(\cdot)\right)=h\left(Y^{2}(\cdot), Z^{2}(\cdot)\right) .
$$

We define $f^{i}(t)=f\left(t, Y^{i}(t), Y_{\delta}^{i}(t), Z^{i}(t)\right)$ and $g^{i}(t)=g\left(t, Y^{i}(t), Y_{\delta}^{i}(t), Z^{i}(t)\right)$ for $i=1,2$. Denoted their difference by

$$
\begin{aligned}
(\widehat{Y}(\cdot), \widehat{Z}(\cdot)): & =\left(Y^{1}(\cdot)-Y^{2}(\cdot), Z^{1}(\cdot)-Z^{2}(\cdot)\right), \\
(\widehat{y}(\cdot), \widehat{z}(\cdot)): & =\left(y^{1}(\cdot)-y^{2}(\cdot), z^{1}(\cdot)-z^{2}(\cdot)\right)
\end{aligned}
$$

and

Then $(\widehat{y}(t), \widehat{z}(t))$ satisfies

$$
\widehat{f}(t):=f^{1}(t)-f^{2}(t), \quad \widehat{g}(t):=g^{1}(t)-g^{2}(t) .
$$

$$
\left\{\begin{aligned}
d \widehat{y}(t) & =\widehat{f}(t) d t+\widehat{g}(t) d \overrightarrow{W(t)}-\widehat{z}(t) d \overleftarrow{B(t)}, \quad t \in[0, T] \\
\widehat{y}(t) & =0, \quad t \in[-\delta, 0]
\end{aligned}\right.
$$

Applying Lemma 2.1 to $e^{-\beta t}\left|\widehat{y}_{t}\right|^{2}$ and taking expectation, we have the following inequality

$$
E \int_{0}^{T} e^{-\beta t}\left(\beta|\widehat{y}(t)|^{2}+|\widehat{z}(t)|^{2}\right) d t \leqslant 2 E \int_{0}^{T} e^{-\beta t} \widehat{y}(t) \cdot \widehat{f}(t) d t+E \int_{0}^{T} e^{-\beta t}|\widehat{g}(t)|^{2} d t
$$

Notice that

$$
2 E \int_{0}^{T} e^{-\beta t} \widehat{y}(t) \cdot \widehat{f}(t) d t \leqslant \frac{\beta}{2} E \int_{0}^{T} e^{-\beta t}|\widehat{y}(t)|^{2} d t+\frac{2}{\beta} E \int_{0}^{T} e^{-\beta t}|\widehat{f}(t)|^{2} d t
$$

From $(\mathrm{H} 2)$, we have

$$
E \int_{0}^{T} e^{-\beta t}|\widehat{f}(t)|^{2} d t \leqslant E \int_{0}^{T} e^{-\beta t} C\left[|\widehat{Y}(t)|^{2}+\left|\widehat{Y}_{\delta}(t)\right|^{2}+|\widehat{Z}(t)|^{2}\right] d t .
$$

From (H3), we know there exists a constant $L>0$, such that

$$
\int_{0}^{T}\left|\widehat{Y}_{\delta}(t)\right|^{2} d t \leqslant \int_{0}^{T} L|\widehat{Y}(t)|^{2} d t
$$

Then the inequality (3.3) can be written as 


$$
E \int_{0}^{T} e^{-\beta t}|\widehat{f}(t)|^{2} d t \leqslant E \int_{0}^{T} e^{-\beta t}\left[C(L+1)|\widehat{Y}(t)|^{2}+C|\widehat{Z}(t)|^{2}\right] d t \leqslant C(L+1) E \int_{0}^{T} e^{-\beta t}\left[|\widehat{Y}(t)|^{2}+|\widehat{Z}(t)|^{2}\right] d t
$$

Similarly, from (H3) and (H4) we have

$$
\begin{aligned}
E \int_{0}^{T} e^{-\beta t}|\widehat{g}(t)|^{2} d t & \leqslant E \int_{0}^{T} e^{-\beta t}\left(C\left[|\widehat{Y}(t)|^{2}+\left|\widehat{Y}_{\delta}(t)\right|^{2}\right]+\alpha|\widehat{Z}(t)|^{2}\right) d t \\
& \leqslant C(L+1) E \int_{0}^{T} e^{-\beta t}|\widehat{Y}(t)|^{2} d t+\alpha E \int_{0}^{T} e^{-\beta t}|\widehat{Z}(t)|^{2} d t
\end{aligned}
$$

Then we have

$$
E \int_{0}^{T} e^{-\beta t}\left(\frac{\beta}{2}|\widehat{y}(t)|^{2}+|\widehat{z}(t)|^{2}\right) d t \leqslant \frac{2}{\beta} C(1+L)\left(\frac{2}{\beta}+1\right) E \int_{0}^{T} \frac{\beta}{2} e^{-\beta t}|\widehat{Y}(t)|^{2} d t+\left(\frac{2 C}{\beta}+\alpha\right) E \int_{0}^{T} e^{-\beta t}|\widehat{Z}(t)|^{2} d t .
$$

We select $\beta=2 C(L+1)+2$, then we have

(1) $\beta^{2}-2 C(L+1) \beta-4 C(L+1)>0, \beta>0$, that is $\frac{2}{\beta} C(1+L)\left(\frac{2}{\beta}+1\right)<1$;

(2) from the assumption $0<\alpha<\frac{\mathrm{L}}{\mathrm{L}+1}$, we have

$$
\frac{2 \mathrm{C}}{\beta}+\alpha<\frac{2 \mathrm{C}}{2 \mathrm{C}(\mathrm{L}+1)+2}+1-\frac{1}{\mathrm{~L}+1}=1+\frac{\mathrm{C}}{\mathrm{C}(\mathrm{L}+1)+1}-\frac{\mathrm{C}}{\mathrm{C}(\mathrm{L}+1)}<1
$$

Then we have the following inequality

$$
E \int_{0}^{T} e^{-\beta t}\left(\frac{\beta}{2}|\widehat{y}(t)|^{2}+|\widehat{z}(t)|^{2}\right) d t<E \int_{0}^{T} e^{-\beta t}\left(\frac{\beta}{2}|\widehat{Y}(t)|^{2}+|\widehat{Z}(t)|^{2}\right) d t
$$

that is

$$
\|(\hat{y}(t), \hat{z}(t))\|_{\beta}<\|(\widehat{Y}(t), \widehat{Z}(t))\|_{\beta} .
$$

We obtain that $h$ is a contraction mapping. Then it follows from the fixed point theorem that the delayed BDSDEs (3.1) has a unique solution. So we complete the proof of Theorem 3.1.

Let $u^{*}(\cdot)$ be an optimal control of the systems. For any $v(\cdot) \in \mathrm{U}[0, \mathrm{~T}]$, let $u^{\varepsilon}(\cdot)=u^{*}(\cdot)+\varepsilon\left(v(\cdot)-u^{*}(\cdot)\right)$. For simplicity, we rewrite $v(t)-u^{*}(t)$ as $v(t)$. Because of the convexity, $u^{\varepsilon}(\cdot)$ is in $u[0, T]$. From Theorem 3.1, we know that state equation (2.1) has a unique solution. Let $\left(y^{*}(\cdot), z^{*}(\cdot)\right)$ and $\left(y^{\varepsilon}(\cdot), z^{\varepsilon}(\cdot)\right)$ are the solutions of (2.1) corresponding to $u^{*}(\cdot)$ and $u^{\varepsilon}(\cdot)$, respectively.

Let $\varphi^{\varepsilon}(\mathrm{t})=\varphi\left(\mathrm{t}, \mathrm{y}^{\varepsilon}(\mathrm{t}), z^{\varepsilon}(\mathrm{t}), \mathrm{u}^{\varepsilon}(\mathrm{t})\right)$ and $\varphi^{*}(\mathrm{t})=\varphi\left(\mathrm{t}, \mathrm{y}^{*}(\mathrm{t}), z^{*}(\mathrm{t}), \mathrm{u}^{*}(\mathrm{t})\right)$, where $\varphi$ denotes $\mathrm{f}, \mathrm{l}, \mathrm{g}$ and their derivative functions. Let us introduce the variational equation as follows:

$$
\left\{\begin{aligned}
d y_{1}(t)= & {\left[f_{y}^{*}(t) y_{1}(t)+f_{y_{\delta}}^{*}(t) y_{1 \delta}(t)+f_{z}^{*}(t) z_{1}(t)+f_{u}^{*}(t) v(t)\right] d t } \\
& +\left[g_{y}^{*}(t) y_{1}(t)+g_{y_{\delta}}^{*}(t) y_{1 \delta}(t)+g_{z}^{*}(t) z_{1}(t)+g_{u}^{*}(t) v(t)\right] d \overrightarrow{W(t)}-z_{1}(t) d \overleftarrow{B(t)}, \quad t \in[0, T], \\
y_{1}(t)= & 0, \quad t \in[-\delta, 0] .
\end{aligned}\right.
$$

Lemma 3.2. Let (H1) hold and

$$
\widetilde{y}^{\varepsilon}(t)=\frac{y^{\varepsilon}(t)-y^{*}(t)}{\varepsilon}-y_{1}(t), \quad \widetilde{z}^{\varepsilon}(t)=\frac{z^{\varepsilon}(t)-z^{*}(t)}{\varepsilon}-z_{1}(t)
$$

Then

$$
\lim _{\varepsilon \rightarrow 0} E\left[\left|\widetilde{y}^{\varepsilon}(t)\right|^{2}\right]=0, \quad \lim _{\varepsilon \rightarrow 0} E \int_{0}^{T}\left\|\tilde{z}^{\varepsilon}(t)\right\|^{2} d t=0 .
$$

Proof. Under the assumption (H1) and Lemma 2.1, using the first order development and Gronwall's inequality we get the conclusion. 
Remark 3.3. The conclusion of Lemma 3.2 is mainly about the estimation of the solution. This is an important lemma to prove our main results. Its proof method is similar to the Lemma 3.3 in Han et al. [6], we will not repeat in this paper.

Lemma 3.4. Assume that (H1) holds. Then we have the following variational inequality

$$
E \int_{0}^{T}\left[l_{y}^{*}(t) y_{1}(t)+l_{z}^{*}(t) z_{1}(t)+l_{u}^{*}(t) v(t)\right] d t+E\left[\Phi_{y}\left(y^{*}(T)\right) y_{1}(T)\right] \leqslant 0
$$

Proof. Let $\mathrm{u}^{*}(\cdot)$ be the optimal control. Then for any $v(\cdot) \in \mathrm{U}[0, \mathrm{~T}]$ and $\mathrm{u}^{\varepsilon}(\cdot) \in \mathrm{U}[0, \mathrm{~T}]$, we have

$$
\mathrm{J}\left(\mathrm{u}^{\varepsilon}(\cdot)\right)-\mathrm{J}\left(\mathrm{u}^{*}(\cdot)\right) \leqslant 0 \text {. }
$$

That is

$$
E \int_{0}^{T}\left[l\left(t, y^{\varepsilon}(t), z^{\varepsilon}(t), u^{\varepsilon}(t)\right)-l\left(t, y^{*}(t), z^{*}(t), u^{*}(t)\right)\right] d t+E\left[\Phi\left(y^{\varepsilon}(T)\right)-\Phi\left(y^{*}(T)\right)\right] \leqslant 0 .
$$

Hence we have

$$
\lim _{\varepsilon \rightarrow 0} \frac{1}{\varepsilon}\left\{E \int_{0}^{T}\left[l\left(t, y^{\varepsilon}(t), z^{\varepsilon}(t), u^{\varepsilon}(t)\right)-l\left(t, y^{*}(t), z^{*}(t), u^{*}(t)\right)\right] d t+E\left[\Phi\left(y^{\varepsilon}(T)\right)-\Phi\left(y^{*}(T)\right)\right]\right\} \leqslant 0 .
$$

We know that

$$
\begin{aligned}
\lim _{\varepsilon \rightarrow 0} & \frac{1}{\varepsilon} E \int_{0}^{T}\left[l\left(t, y^{\varepsilon}(t), z^{\varepsilon}(t), u^{\varepsilon}(t)\right)-l\left(t, y^{*}(t), z^{*}(t), u^{*}(t)\right)\right] d t \\
= & \lim _{\varepsilon \rightarrow 0} \frac{1}{\varepsilon} E \int_{0}^{T}\left\{\left[l\left(t, y^{\varepsilon}(t), z^{\varepsilon}(t), u^{\varepsilon}(t)\right)-l\left(t, y^{*}(t), z^{\varepsilon}(t), u^{\varepsilon}(t)\right)\right]\right. \\
& +\left[l\left(t, y^{*}(t), z^{\varepsilon}(t), u^{\varepsilon}(t)\right)-l\left(t, y^{*}(t), z^{*}(t), u^{\varepsilon}(t)\right)\right] \\
& \left.+\left[l\left(t, y^{*}(t), z^{*}(t), u^{\varepsilon}(t)\right)-l\left(t, y^{*}(t), z^{*}(t), u^{*}(t)\right)\right]\right\} d t .
\end{aligned}
$$

Notice that $\mathfrak{u}^{\varepsilon}(\mathrm{t})=\mathrm{u}^{*}(\mathrm{t})+\varepsilon v(t)$ and by Lemma 3.2, we have

$$
\begin{aligned}
\lim _{\varepsilon \rightarrow 0} & \frac{1}{\varepsilon} E \int_{0}^{T}\left[l\left(t, y^{\varepsilon}(t), z^{\varepsilon}(t), u^{\varepsilon}(t)\right)-l\left(t, y^{*}(t), z^{*}(t), u^{*}(t)\right)\right] d t \\
& =E \int_{0}^{T}\left[l_{y}^{*}(t) y_{1}(t)+l_{z}^{*}(t) z_{1}(t)+l_{u}^{*}(t) v(t)\right] d t .
\end{aligned}
$$

Moreover,

$$
\lim _{\varepsilon \rightarrow 0} \frac{1}{\varepsilon} E\left[\Phi\left(y^{\varepsilon}(T)\right)-\Phi\left(y^{*}(T)\right)\right]=\lim _{\varepsilon \rightarrow 0} E\left[\frac{\Phi\left(y^{\varepsilon}(T)\right)-\Phi\left(y^{*}(T)\right)}{y^{\varepsilon}(T)-y^{*}(T)} \cdot \frac{y^{\varepsilon}(T)-y^{*}(T)}{\varepsilon}\right]=E\left[\Phi_{y}\left(y^{*}(T)\right) y_{1}(T)\right] .
$$

So we have the variational inequality

$$
E \int_{0}^{T}\left[l_{y}^{*}(t) y_{1}(t)+l_{z}^{*}(t) z_{1}(t)+l_{u}^{*}(t) v(t)\right] d t+E\left[\Phi_{y}\left(y^{*}(T) y_{1}(T)\right)\right] \leqslant 0
$$

Theorem 3.5. Let $\left(\mathrm{y}^{*}(\cdot), z^{*}(\cdot), \mathrm{u}^{*}(\cdot)\right)$ be an optimal triple of the problem (2.1)-(2.2). If the time delay $\delta$ is sufficiently small, there is a unique $\mathcal{F}_{\mathrm{t}}$-adapted solution satisfying the associated adjoint equation

$$
\left\{\begin{aligned}
-\mathrm{d} p(t)= & \left\{\left(f_{y}^{*}(t)\right)^{\top} p(t)+E^{\mathcal{F}_{t}}\left[\left(f_{y_{\delta}}^{*}(t+\delta)\right)^{\top} p(t+\delta)\right]+E^{\mathcal{F}_{t}}\left[\left(g_{y_{\delta}}^{*}(t+\delta)\right)^{\top} q(t+\delta)\right]+\left(g_{y}^{*}(t)\right)^{\top} q(t)\right. \\
& \left.-l_{y}^{*}(t)\right\} d t+\left[l_{z}^{*}(t)-\left(f_{z}^{*}(t)\right)^{\top} p(t)-\left(g_{z}^{*}(t)\right)^{\top} q(t)\right] d \overleftrightarrow{B(t)}-q(t) d \overrightarrow{W(t)}, t \in[0, T], \\
p(T)= & -\Phi_{y}\left(y^{*}(T)\right), \\
p(t)= & 0, \quad t \in(T, T+\delta], \\
q(t)= & 0, \quad t \in(T, T+\delta],
\end{aligned}\right.
$$

and with probability 1 , we have 


$$
\left\langle H_{u}\left(t, y^{*}(t), y_{\mathcal{\delta}}^{*}(t), z^{*}(t), u^{*}(t), p(t), q(t)\right), v(t)-u^{*}(t)\right\rangle \geqslant 0,
$$

for a.e. $\mathrm{t} \in[0, \mathrm{~T}], \forall v(\cdot) \in \mathrm{U}[0, \mathrm{~T}]$, where

$$
\begin{aligned}
& H\left(t, y(t), y_{\delta}(t), z(t), u(t), p(t), q(t)\right) \\
& =f\left(t, y(t), y_{\delta}(t), z(t), u(t)\right)^{\top} p(t)+g\left(t, y(t), y_{\delta}(t), z(t), u(t)\right)^{\top} q(t)-l(t, y(t), z(t), u(t)), \\
& \quad\left(t, y(t), y_{\delta}(t), z(t), v(t), p(t), q(t)\right) \in[0, T] \times R^{n} \times R^{n} \times R^{n \times m} \times U \times R^{n} \times R^{n \times d} .
\end{aligned}
$$

Proof. We can see the equation (3.4) is an anticipated BDSDE. The existence and uniqueness of the solution for the equations (3.4) can be guaranteed by Theorem 3.2 in [17] and Theorem 2.4 in [19]. Applying Itô's formula to $\left\langle p(t), y_{1}(t)\right\rangle$, we have

$$
\left\langle p(T), y_{1}(T)\right\rangle-\left\langle p(0), y_{1}(0)\right\rangle=\int_{0}^{T} p(t) d y_{1}(t)+d p(t) y_{1}(t)+d p(t) d y_{1}(t)
$$

Taking the expectation, we have that

$$
\begin{aligned}
E\left\langle p(T), y_{1}(T)\right\rangle= & E \int_{0}^{T}\left\{\left\langle p(t), f_{y}^{*}(t) y_{1}(t)+f_{y_{\delta}}^{*}(t) y_{1 \delta}(t)+f_{z}^{*}(t) z_{1}(t)+f_{u}^{*}(t) v(t)\right\rangle\right. \\
& +\left\langle-\left(f_{y}^{*}(t)\right)^{\top} p(t)-E^{\mathcal{F}_{t}}\left[\left(f_{y_{\delta}}^{*}(t+\delta)\right)^{\top} p(t+\delta)\right]-E^{\mathcal{F}_{t}}\left[\left(g_{y_{\delta}}^{*}(t+\delta)\right)^{\top} q(t+\delta)\right]-\left(g_{y}^{*}(t)\right)^{\top} q(t)\right. \\
& \left.+l_{y}^{*}(t), y_{1}(t)\right\rangle+\left\langle q(t), g_{y}^{*}(t) y_{1}(t)+g_{y_{\delta}}^{*}(t) y_{1 \delta}(t)+g_{z}^{*}(t) z_{1}(t)+g_{u}^{*}(t) v(t)\right\rangle \\
& \left.+\left\langle\left(f_{z}^{*}(t)\right)^{\top} p(t)+\left(g_{z}^{*}(t)\right)^{\top} q(t)-l_{z}^{*}(t),-z_{1}(t)\right\rangle\right\} d t .
\end{aligned}
$$

Noticing the initial and terminal conditions, we obtain

$$
E\left\{\int_{0}^{T}\left\langle p(t),-f_{y_{\delta}}^{*}(t) y_{1 \delta}(t)\right\rangle+\left\langle E^{\mathcal{F}_{t}}\left[\left(f_{y_{\delta}}^{*}(t+\delta)\right)^{\top} p(t+\delta)\right], y_{1}(t)\right\rangle d t\right\}=0
$$

and

$$
E\left\{\int_{0}^{T}\left\langle q(t),-g_{y_{\delta}}^{*}(t) y_{1 \delta}(t)\right\rangle+\left\langle E^{\mathcal{F}_{t}}\left[\left(g_{y_{\delta}}^{*}(t+\delta)\right)^{\top} q(t+\delta)\right], y_{1}(t)\right\rangle d t\right\}=0
$$

These show

$$
E\left\langle p(T), y_{1}(T)\right\rangle=E \int_{0}^{T}\left[\left\langle p(t), f_{u}^{*}(t) v(t)\right\rangle+\left\langle q(t), g_{u}^{*}(t) v(t)\right\rangle+\left\langle l_{y}^{*}(t), y_{1}(t)\right\rangle+\left\langle l_{z}^{*}(t), z_{1}(t)\right\rangle\right] d t,
$$

that is,

$$
-E\left\langle\Phi_{y}\left(y^{*}(T)\right), y_{1}(T)\right\rangle=E \int_{0}^{T}\left[\left\langle p(t), f_{u}^{*}(t) v(t)\right\rangle+\left\langle q(t), g_{u}^{*}(t) v(t)\right\rangle+\left\langle l_{y}^{*}(t), y_{1}(t)\right\rangle+\left\langle l_{z}^{*}(t), z_{1}(t)\right\rangle\right] d t .
$$

Hence,

$$
\begin{aligned}
E & \left.=\Phi_{y}\left(y^{*}(T)\right), y_{1}(T)\right\rangle+E \int_{0}^{T}\left[\left\langle l_{y}^{*}(t), y_{1}(t)\right\rangle+\left\langle l_{z}^{*}(t), z_{1}(t)\right\rangle+\left\langle l_{u}^{*}(t), v(t)\right\rangle\right] d t \\
& =E \int_{0}^{T}\left\langle l_{u}^{*}(t)-g_{u}^{*}(t) q(t)-f_{u}^{*}(t) p(t), v(t)\right\rangle d t \\
& =E \int_{0}^{T}\left\langle-H_{u}\left(t, y^{*}(t), y_{\delta}^{*}(t), z^{*}(t), u^{*}(t), p(t), q(t)\right), v(t)\right\rangle d t .
\end{aligned}
$$


From Lemma 3.4 we have

$$
E \int_{0}^{T}\left[l_{y}^{*}(t) y_{1}(t)+l_{z}^{*}(t) z_{1}(t)+l_{u}^{*}(t) v(t)\right] d t+E\left[\Phi_{y}\left(y^{*}(T)\right) y_{1}(T)\right] \leqslant 0,
$$

that is,

$$
E \int_{0}^{T}\left\langle H_{u}\left(t, y^{*}(t), y_{\delta}^{*}(t), z^{*}(t), u^{*}(t), p(t), q(t)\right), v(t)\right\rangle \geqslant 0
$$

So for any $v \in \mathrm{U}[0, \mathrm{~T}]$ and for all $\mathrm{A} \in \mathcal{F}_{\mathrm{t}}$,

$$
E\left[1_{A}\left\langle H_{u}\left(t, y^{*}(t), y_{\delta}^{*}(t), z^{*}(t), u^{*}(t), p(t), q(t)\right), v(t)-u^{*}(t)\right\rangle\right] \geqslant 0 .
$$

We complete the proof.

Next we will give the sufficient condition of optimality under certain assumptions.

Theorem 3.6. Suppose $\mathrm{u}^{*}(\cdot) \in \mathrm{U}[0, \mathrm{~T}]$. Let $\left(\mathrm{y}^{*}(\cdot), z^{*}(\cdot)\right)$ and $\mathrm{p}(\cdot)$ be the corresponding solutions of (2.1) and (3.4), respectively. Suppose the following assumptions hold:

(H5) $\mathrm{H}(\mathrm{t}, \cdot, \cdot, \cdot, \cdot, \mathrm{p}(\mathrm{t}), \mathrm{q}(\mathrm{t}))$ is a concave function and $\Phi(\cdot)$ is a convex function for all $\mathrm{t} \in[0, \mathrm{~T}]$;

(H6) $\mathrm{H}\left(\mathrm{t}, \mathrm{y}^{*}(\mathrm{t}), \mathrm{y}_{\mathcal{\delta}}^{*}(\mathrm{t}), \mathrm{z}^{*}(\mathrm{t}), \mathrm{u}^{*}(\mathrm{t}), \mathrm{p}(\mathrm{t}), \mathrm{q}(\mathrm{t})\right)=\sup _{v \in \mathrm{U}[0, \mathrm{~T}]} \mathrm{H}\left(\mathrm{t}, \mathrm{y}(\mathrm{t}), \mathrm{y}_{\delta}(\mathrm{t}), z(\mathrm{t}), v(\mathrm{t}), \mathrm{p}(\mathrm{t}), \mathrm{q}(\mathrm{t})\right)$.

Then $\mathrm{u}^{*}(\cdot)$ is an optimal control for delayed doubly stochastic optimal problems (2.1)-(2.2).

Proof. For any $v(\cdot) \in \mathrm{U}[0, \mathrm{~T}]$, let $(\mathrm{y}(\cdot), z(\cdot))$ be the corresponding trajectory.

Let

$$
\begin{aligned}
& J_{1}=E \int_{0}^{T}\left[l\left(t, y^{*}(t), z^{*}(t), u^{*}(t)\right)-l(t, y(t), z(t), v(t))\right] d t \\
& J_{2}=E\left[\Phi\left(y^{*}(T)\right)-\Phi(y(T))\right]
\end{aligned}
$$

We want to prove

$$
\mathrm{J}\left(\mathrm{u}^{*}\right)-\mathrm{J}(v)=\mathrm{J}_{1}+\mathrm{J}_{2} \geqslant 0
$$

Since $\Phi(\cdot)$ is convex, we have

$$
\mathrm{J}_{2}=\mathrm{E}\left[\Phi\left(\mathrm{y}^{*}(\mathrm{~T})\right)-\Phi(\mathrm{y}(\mathrm{T}))\right] \geqslant \mathrm{E}\left[\Phi_{\mathrm{y}}^{\top}\left(\mathrm{y}^{*}(\mathrm{~T})\right)\left(\mathrm{y}^{*}(\mathrm{~T})-\mathrm{y}(\mathrm{T})\right)\right]=\mathrm{E}\left[-\mathrm{p}^{\top}(\mathrm{T})\left(\mathrm{y}^{*}(\mathrm{~T})-\mathrm{y}(\mathrm{T})\right)\right] .
$$

Applying Itô's formula to $\left\langle p(\cdot), y^{*}(\cdot)-y(\cdot)\right\rangle$ and taking expectation, we have

$$
\begin{aligned}
& E\left[p^{\top}(T)\left(y^{*}(T)-y(T)\right)\right] \\
&=E \int_{0}^{T} d p(t)\left(y^{*}(t)-y(t)\right)+E \int_{0}^{T} p(t) d\left(y^{*}(t)-y(t)\right)+E \int_{0}^{T} d p(t) d\left(y^{*}(t)-y(t)\right) \\
&=E \int_{0}^{T}\left\langle-\left(f_{y}^{*}(t)\right)^{\top} p(t)-E^{\mathcal{F}_{t}}\left[\left(f_{y_{\delta}}^{*}(t+\delta)\right)^{\top} p(t+\delta)\right]-E^{\mathcal{F}_{t}}\left[\left(g_{y_{\delta}}^{*}(t+\delta)\right)^{\top} q(t+\delta)\right]-\left(g_{y}^{*}(t)\right)^{\top} q(t)\right. \\
&\left.+l_{y}^{*}(t), y^{*}(t)-y(t)\right\rangle d t+E \int_{0}^{T}\left\langle p(t), f\left(t, y^{*}(t), y_{\delta}^{*}(t), z^{*}(t), u^{*}(t)\right)-f\left(t, y(t), y_{\delta}(t), z(t), v(t)\right)\right\rangle d t \\
&+E \int_{0}^{T}\left\langle q(t), g\left(t, y^{*}(t), y_{\delta}^{*}(t), z^{*}(t), u^{*}(t)\right)-g\left(t, y(t), y_{\delta}(t), z(t), v(t)\right)\right\rangle d t \\
&+E \int_{0}^{T}\left\langle l_{z}^{*}(t)-\left(f_{z}^{*}(t)\right)^{\top} p(t)-\left(g_{z}^{*}(t)\right)^{\top} q(t), z^{*}(t)-z(t)\right\rangle d t .
\end{aligned}
$$


From the definition of the Hamiltonian function, we have

$$
\begin{aligned}
H_{y}^{*} & =\left(f_{y}^{*}(t)\right)^{\top} p(t)+\left(g_{y}^{*}(t)\right)^{\top} q(t)-l_{y}^{*}(t), \\
H_{y_{\delta}}^{*} & =\left(f_{y_{\delta}}^{*}(t)\right)^{\top} p(t)+\left(g_{y \delta}^{*}(t)\right)^{\top} q(t), \\
H_{z}^{*} & =\left(f_{z}^{*}(t)\right)^{\top} p(t)+\left(g_{z}^{*}(t)\right)^{\top} q(t)-l_{z}^{*}(t) .
\end{aligned}
$$

Then

$$
\begin{aligned}
J_{2} \geqslant & E\left[-p^{\top}(T)\left(y^{*}(T)-y(T)\right)\right] \\
= & E \int_{0}^{T}\left\langle H_{y}^{*}\left(t, y^{*}(t), y_{\delta}^{*}(t), z^{*}(t), u^{*}(t), p(t), q(t)\right), y^{*}(t)-y(t)\right\rangle d t \\
& +E \int_{0}^{T}\left\langle E^{\mathcal{F}_{t}}\left[\left(f_{y_{\delta}}^{*}(t+\delta)\right)^{\top} p(t+\delta)\right]+E^{\mathcal{F}_{t}}\left[\left(g_{y_{\delta}}^{*}(t+\delta)\right)^{\top} q(t+\delta)\right], y^{*}(t)-y(t)\right\rangle d t \\
& +E \int_{0}^{T}\left\langle p(t),-f\left(t, y^{*}(t), y_{\delta}^{*}(t), z^{*}(t), u^{*}(t)\right)+f\left(t, y(t), y_{\delta}(t), z(t), v(t)\right)\right\rangle d t \\
& +E \int_{0}^{T}\left\langle q(t),-g\left(t, y^{*}(t), y_{\delta}^{*}(t), z^{*}(t), u^{*}(t)\right)+g\left(t, y(t), y_{\delta}(t), z(t), v(t)\right)\right\rangle d t \\
& +E \int_{0}^{T}\left\langle H_{z}^{*}\left(t, y^{*}(t), y_{\delta}^{*}(t), z^{*}(t), u^{*}(t), p(t), q(t)\right), z^{*}(t)-z(t)\right\rangle d t, \\
J_{1}= & E \int_{0}^{T}\left[l\left(t, y^{*}(t), z^{*}(t), u^{*}(t)\right)-l(t, y(t), z(t), v(t))\right] d t \\
= & E \int_{0}^{T}\left[-H\left(t, y^{*}(t), y_{\delta}^{*}(t), z^{*}(t), u^{*}(t), p(t), q(t)\right)+H(t, y(t), y \delta(t), z(t), v(t), p(t), q(t))\right] d t \\
& +E \int_{0}^{T}\left\langle g\left(t, y^{*}(t), y_{\delta}^{*}(t), z^{*}(t), u^{*}(t)\right)-g(t, y(t), y(t), z(t), v(t)), q(t)\right\rangle d t \\
& +E \int_{0}^{T}\left\langle f\left(t, y^{*}(t), y_{\delta}^{*}(t), z^{*}(t), u^{*}(t)\right)-f(t, y(t), y \delta(t), z(t), v(t)), p(t)\right\rangle d t .
\end{aligned}
$$

So we have

$$
\begin{aligned}
J_{1}+J_{2} \geqslant & E \int_{0}^{T}\left\langle H_{y}^{*}\left(t, y^{*}(t), y_{\delta}^{*}(t), z^{*}(t), u^{*}(t), p(t), q(t)\right), y^{*}(t)-y(t)\right\rangle d t \\
& +E \int_{0}^{T}\left\langle E^{\mathcal{F}_{t}}\left[\left(f_{y_{\delta}}^{*}(t+\delta)\right)^{\top} p(t+\delta)\right]+E^{\mathcal{F}_{t}}\left[\left(g_{y_{\delta}}^{*}(t+\delta)\right)^{\top} q(t+\delta)\right], y^{*}(t)-y(t)\right\rangle d t \\
& +E \int_{0}^{T}\left\langle H_{z}^{*}\left(t, y^{*}(t), y_{\delta}^{*}(t), z^{*}(t), u^{*}(t), p(t), q(t)\right), z^{*}(t)-z(t)\right\rangle d t \\
& -E \int_{0}^{T}\left[H\left(t, y^{*}(t), y_{\delta}^{*}(t), z^{*}(t), u^{*}(t), p(t), q(t)\right)-H\left(t, y(t), y_{\delta}(t), z(t), v(t), p(t), q(t)\right)\right] d t
\end{aligned}
$$

Notice that

$$
\begin{aligned}
E \int_{0}^{T}\left\langle-H_{y_{\delta}}^{*}\left(t, y^{*}(t), y_{\delta}^{*}(t), z^{*}(t), u^{*}(t), p(t), q(t)\right), y_{\delta}^{*}(t)-y_{\delta}(t)\right\rangle d t \\
\quad+E \int_{0}^{T}\left\langle E^{\mathcal{F}_{t}}\left[\left(f_{y_{\delta}}^{*}(t+\delta)\right)^{\top} p(t+\delta)\right]+E^{\mathcal{F}_{t}}\left[\left(g_{y_{\delta}}^{*}(t+\delta)\right)^{\top} q(t+\delta)\right], y^{*}(t)-y(t)\right\rangle d t \\
=E \int_{0}^{T}\left\langle-\left(g_{y_{\delta}}^{*}(t)\right)^{\top} q(t)-\left(f_{y_{\delta}}^{*}(t)\right)^{\top} p(t), y_{\delta}^{*}(t)-y_{\delta}(t)\right\rangle d t
\end{aligned}
$$




$$
\begin{aligned}
& +E \int_{0}^{T}\left\langle E^{\mathcal{F}_{t}}\left[\left(f_{y_{\delta}}^{*}(t+\delta)\right)^{\top} p(t+\delta)\right]+E^{\mathcal{F}_{t}}\left[\left(g_{y_{\delta}}^{*}(t+\delta)\right)^{\top} q(t+\delta)\right], y^{*}(t)-y(t)\right\rangle d t \\
= & E \int_{0}^{T}\left\langle-\left(f_{y_{\delta}}^{*}(t)\right)^{\top} p(t), y_{\delta}^{*}(t)-y_{\delta}(t)\right\rangle d t+E \int_{0}^{T}\left\langle E^{\mathcal{F}_{t}}\left[\left(f_{y_{\delta}}^{*}(t+\delta)\right)^{\top} p(t+\delta)\right], y^{*}(t)-y(t)\right\rangle d t \\
& +E \int_{0}^{T}\left\langle-\left(g_{y_{\delta}}^{*}(t)\right)^{\top} q(t), y_{\delta}^{*}(t)-y_{\delta}(t)\right\rangle d t+E \int_{0}^{T}\left\langle E^{\mathcal{F}_{t}}\left[\left(g_{y_{\delta}}^{*}(t+\delta)\right)^{\top} q(t+\delta)\right], y^{*}(t)-y(t)\right\rangle d t \\
= & 0 .
\end{aligned}
$$

Then

$$
\begin{aligned}
J_{1}+J_{2} \geqslant & E \int_{0}^{T}\left[H\left(t, y(t), y_{\delta}(t), z(t), v(t), p(t), q(t)\right)-H\left(t, y^{*}(t), y_{\delta}^{*}(t), z^{*}(t), u^{*}(t), p(t), q(t)\right)\right] d t \\
& +E \int_{0}^{T}\left\langle H_{y}^{*}\left(t, y^{*}(t), y_{\delta}^{*}(t), z^{*}(t), u^{*}(t), p(t), q(t)\right), y^{*}(t)-y(t)\right\rangle d t \\
& +E \int_{0}^{T}\left\langle H_{y_{\delta}}^{*}\left(t, y^{*}(t), y_{\delta}^{*}(t), z^{*}(t), u^{*}(t), p(t), q(t)\right), y_{\delta}^{*}(t)-y_{\delta}(t)\right\rangle d t \\
& +E \int_{0}^{T}\left\langle H_{z}^{*}\left(t, y^{*}(t), y_{\delta}^{*}(t), z^{*}(t), u^{*}(t), p(t), q(t)\right), z^{*}(t)-z(t)\right\rangle d t .
\end{aligned}
$$

Since $\left(y(t), y_{\delta}(t), z(t), v(t)\right) \rightarrow H\left(y(t), y_{\delta}(t), z(t), v(t)\right)$ is concave, we have

$$
\begin{aligned}
& H\left(t, y(t), y_{\delta}(t), z(t), v(t), p(t), q(t)\right)-H\left(t, y^{*}(t), y_{\delta}^{*}(t), z^{*}(t), u^{*}(t), p(t), q(t)\right) \\
& \geqslant\left\langle H_{y}^{*}\left(t, y^{*}(t), y_{\delta}^{*}(t), z^{*}(t), u^{*}(t), p(t), q(t)\right), y(t)-y^{*}(t)\right\rangle \\
&+\left\langle H_{y_{\delta}}^{*}\left(t, y^{*}(t), y_{\delta}^{*}(t), z^{*}(t), u^{*}(t), p(t), q(t)\right), y_{\delta}(t)-y_{\delta}^{*}(t)\right\rangle \\
&+\left\langle H_{z}^{*}\left(t, y^{*}(t), y_{\delta}^{*}(t), z^{*}(t), u^{*}(t), p(t), q(t)\right), z(t)-z^{*}(t)\right\rangle \\
&+\left\langle H_{u}^{*}\left(t, y^{*}(t), y_{\delta}^{*}(t), z^{*}(t), u^{*}(t), p(t), q(t)\right), v(t)-u^{*}(t)\right\rangle .
\end{aligned}
$$

From the assumption (H6), we obtain

$$
\mathrm{J}_{1}+\mathrm{J}_{2} \geqslant 0
$$

Then we have proved

$$
\mathrm{J}\left(\mathrm{u}^{*}\right)-\mathrm{J}(v)=\mathrm{J}_{1}+\mathrm{J}_{2} \geqslant 0 .
$$

Since $v(\cdot) \in \mathrm{U}[0, \mathrm{~T}]$ is arbitrary, this proves that $\mathrm{u}^{*}(\cdot)$ is optimal.

\section{Applications in LQ problems}

In this section, we apply our maximal principle (Theorem 3.5) to delayed doubly stochastic LQ problem. Assume that

$$
\begin{aligned}
f\left(t, y(t), y_{\delta}(t), z(t), u(t)\right) & =A(t) y(t)+B(t) y_{\delta}(t)+C(t) z(t)+D(t) u(t), \\
g\left(t, y(t), y_{\delta}(t), z(t), u(t)\right) & =F(t) y(t)+G(t) y_{\delta}(t)+M(t) z(t)+N(t) u(t), \\
l\left(t, y(t), y_{\delta}(t), z(t), u(t)\right) & =\frac{1}{2} K(t) y(t) \cdot y(t)+\frac{1}{2} R(t) z(t) \cdot z(t)+\frac{1}{2} S(t) u(t) \cdot u(t), \\
\Phi(y(t)) & =\frac{1}{2} Q y(t) \cdot y(t),
\end{aligned}
$$

where all functions of $t$ are bounded, $K(t), R(t), Q$ are symmetric nonnegative definite and $S(t)$ is symmetric uniformly positive definite. Then the state equation becomes the following delayed doubly stochastic 
differential equation

$$
\left\{\begin{aligned}
d y(t)= & {\left[A(t) y(t)+B(t) y_{\delta}(t)+C(t) z(t)+D(t) u(t)\right] d t } \\
& +\left[F(t) y(t)+G(t) y_{\delta}(t)+M(t) z(t)+N(t) u(t)\right] d \overrightarrow{W(t)}-z(t) d \overleftarrow{B(t)}, \quad t \in[0, T], \\
y(t)= & \varphi(t), \quad t \in[-\delta, 0]
\end{aligned}\right.
$$

with the cost functional

$$
J(u(\cdot))=E\left\{\int_{0}^{T}\left[\frac{1}{2} K(t) y(t) \cdot y(t)+\frac{1}{2} R(t) z(t) \cdot z(t)+\frac{1}{2} S(t) u(t) \cdot u(t)\right] d t+\Phi(y(T))\right\} .
$$

Based on Theorem 3.5, the corresponding adjoint equation becomes

$$
\left\{\begin{aligned}
-\mathrm{dp}(\mathrm{t})= & \left\{(A(t))^{\top} p(t)+E^{\mathcal{F}_{t}}\left[(\mathrm{~B}(\mathrm{t}+\delta))^{\top} p(t+\delta)\right]+E^{\mathcal{F}_{t}}\left[(\mathrm{G}(\mathrm{t}+\delta))^{\top} q(t+\delta)\right]+(F(t))^{\top} q(t)\right. \\
& -K(t) y(t)\} d t+\left[R(t) z(t)-(C(t))^{\top} p(t)-(M(t))^{\top} q(t)\right] d \overleftarrow{B(t)}-q(t) d \overrightarrow{W(t)}, t \in[0, T], \\
p(T)= & -Q y^{*}(T), \\
p(t)= & 0, \quad t \in(T, T+\delta], \\
q(t)= & 0, \quad t \in(T, T+\delta] .
\end{aligned}\right.
$$

From the definition of the Hamiltonian function in Theorem 3.5, we have

$$
\begin{aligned}
H\left(t, y(t), y_{\delta}(t), z(t), u(t), p(t), q(t)\right)= & \left\langle A(t) y(t)+B(t) y_{\delta}(t)+C(t) z(t)+D(t) u(t), p(t)\right\rangle \\
& +\left\langle F(t) y(t)+G(t) y_{\delta}(t)+M(t) z(t)+N(t) u(t), q(t)\right\rangle \\
& -\frac{1}{2} K(t) y(t) \cdot y(t)-\frac{1}{2} R(t) z(t) \cdot z(t)-\frac{1}{2} S(t) u(t) \cdot u(t) .
\end{aligned}
$$

$\operatorname{Let}\left(y^{*}(t), z^{*}(t), u^{*}(t)\right)$ be the optimal triple. Assume that the anticipated backward doubly stochastic differential equation (4.1) has a unique solution and denote it by $(\mathrm{p}(\mathrm{t}), \mathrm{q}(\mathrm{t}))$. If we assume that $\mathrm{U}[0, \mathrm{~T}]=$ $M^{2}\left([0, T], R^{n}\right)$, we have

$$
\mathrm{H}_{\mathrm{u}}\left(\mathrm{t}, \mathrm{y}^{*}(\mathrm{t}), \mathrm{y}_{\mathcal{\delta}}^{*}(\mathrm{t}), \mathrm{z}^{*}(\mathrm{t}), \mathrm{u}^{*}(\mathrm{t}), \mathrm{p}(\mathrm{t}), \mathrm{q}(\mathrm{t})\right)=0 .
$$

In fact, from the conclusion of Theorem 3.5, we have

$$
\left\langle\mathrm{H}_{\mathrm{u}}\left(\mathrm{t}, \mathrm{y}^{*}(\mathrm{t}), \mathrm{y}_{\delta}^{*}(\mathrm{t}), \mathrm{z}^{*}(\mathrm{t}), \mathrm{u}^{*}(\mathrm{t}), \mathrm{p}(\mathrm{t}), \mathrm{q}(\mathrm{t})\right), v(\mathrm{t})-\mathrm{u}^{*}(\mathrm{t})\right\rangle \geqslant 0, \text { a.e. } \mathrm{t} \in[0, \mathrm{~T}], \forall v(\cdot) \in \mathrm{U}[0, \mathrm{~T}] .
$$

Let $\overline{v(t)}=-v(t)+2 u^{*}(t)$. Notice $\overline{v(t)}$ is also an admission control. From Theorem 3.5, we also have

$$
\left\langle\mathrm{H}_{\mathrm{u}}\left(\mathrm{t}, \mathrm{y}^{*}(\mathrm{t}), \mathrm{y}_{\delta}^{*}(\mathrm{t}), \mathrm{z}^{*}(\mathrm{t}), \mathrm{u}^{*}(\mathrm{t}), \mathrm{p}(\mathrm{t}), \mathrm{q}(\mathrm{t})\right), \overline{v(t)}-\mathrm{u}^{*}(\mathrm{t})\right\rangle \geqslant 0,
$$

that is,

which shows

$$
\left\langle\mathrm{H}_{\mathfrak{u}}\left(\mathrm{t}, \mathrm{y}^{*}(\mathrm{t}), \mathrm{y}_{\delta}^{*}(\mathrm{t}), \mathrm{z}^{*}(\mathrm{t}), \mathrm{u}^{*}(\mathrm{t}), \mathrm{p}(\mathrm{t}), \mathrm{q}(\mathrm{t})\right), v(\mathrm{t})-\mathrm{u}^{*}(\mathrm{t})\right\rangle \leqslant 0,
$$

$$
H_{u}\left(t, y^{*}(t), y_{\delta}^{*}(t), z^{*}(t), u^{*}(t), p(t), q(t)\right)=0
$$

Hence we have

$$
H_{u}\left(t, y^{*}(t), y_{\delta}^{*}(t), z^{*}(t), u^{*}(t), p(t), q(t)\right)=\langle N(t), q(t)\rangle+\langle D(t), p(t)\rangle-S(t) u^{*}(t)=0,
$$

that is,

$$
u^{*}(t)=(S(t))^{-1}\left[(N(t))^{\top} q(t)+(D(t))^{\top} p(t)\right],
$$

where $(p(t), q(t))$ is the solution of the adjoint equation (4.1).

Remark 4.1. In this section, we apply our maximal principle to delayed doubly stochastic LQ problem. From the Theorem 3.5, we give the explicit expression of the optimal control for the delayed LQ problem under some assumptions. But the existence of solutions for the adjoint equation is an important prerequisite. The explicit expression contains the solution of the adjoint equation, which is the anticipated backward doubly stochastic differential equation. This is the main difference with classical maximum principle. 


\section{Conclusions}

In this paper, we have discussed one kind of optimal control problems of time delayed BDSDEs. We established the existence theorem for the delayed BDSDEs and sufficient and necessary conditions for optimal control of this problem. For the future work, we will go on studying this topic, and pay attention to the problems that more variables contains time delay. We will try to extend the current work to much more broad scope of FBDSDE and constantly improve our results in future studies.

\section{Acknowledgment}

The authors thank very much to reviewers for their careful work and thoughtful suggestions that have helped improve this paper substantially. This research was partially supported by China Automobile Industry Innovation and Development Joint Fund U1564213, NSFC Grant 11371169.

\section{References}

[1] L. Chen, J.-H. Huang, Stochastic maximum principle for controlled backward delayed system via advanced stochastic differential equation, J. Optim. Theory Appl., 167 (2015), 1112-1135. 1

[2] L. Chen, Z. Wu, Maximum principle for the stochastic optimal control problem with delay and application, Automatica J. IFAC, 46 (2010), 1074-1080. 1

[3] M. Hafayed, Singular mean-field optimal control for forward-backward stochastic systems and applications to finance, Int. J. Dyn. Control, 2 (2014), 542-554. 1

[4] M. Hafayed, M. Ghebouli, S. Boukaf, Partial information optimal control of mean-field forwardbackward stochastic system driven by Teugels martingales with applications, Neurocomputing, 200 (2016), 11-21.

[5] M. Hafayed, M. Tabet, S. Boukaf, Mean-field maximum principle for optimal control of forward-backward stochastic systems with jumps and its application to mean-variance portfolio problem, Commun. Math. Stat., 3 (2015), 163-186. 1

[6] Y.-C. Han, S.-G. Peng, Z. Wu, Maximum principle for backward doubly stochastic control systems with applications, SIAM J. Control Optim., 48 (2010), 542-554. 1, 3.3

[7] J.-H. Huang, X. Li, J.-T. Shi, Forward-backward linear quadratic stochastic optimal control problem with delay, Systems Control Lett., 61 (2012), 623-630. 1

[8] Q. Lin, A generalized existence theorem of backward doubly stochastic differential equations, Acta Math. Sin. (Engl. Ser.), 26 (2010), 1525-1534. 1

[9] A. Matoussi, M. Scheutzow, Stochastic PDEs driven by nonlinear noise and backward doubly SDEs, J. Theoret. Probab., 15 (2002), 1-39. 1

[10] D. Nualart, É. Pardoux, Stochastic calculus with anticipating integrands, Probab. Theory Related Fields, 78 (1988), 535-581. 2

[11] É. Pardoux, S.-G. Peng, Backward doubly stochastic differential equations and systems of quasilinear SPDEs, Probab. Theory Related Fields, 98 (1994), 209-227. 1, 2, 2.1, 3

[12] S.-G. Peng, Y.-F. Shi, A type of time-symmetric forward-backward stochastic differential equations, C. R. Math. Acad. Sci. Paris, 336 (2003), 773-778. 3

[13] S.-G. Peng, Z. Yang, Anticipated backward stochastic differential equations, Ann. Probab., 37 (2009), 877-902. 1

[14] D. Revuz, M. Yor, Continuous martingales and Brownian motion, Grundlehren der Mathematischen Wissenschaften [Fundamental Principles of Mathematical Sciences], Springer-Verlag, Berlin, (1991). 2

[15] Y.-F. Shi, Y.-L. Gu, K. Liu, Comparison theorems of backward doubly stochastic differential equations and applications, Stoch. Anal. Appl., 23 (2005), 97-110. 1

[16] S. Wu, G.-C. Wang, Optimal control problem of backward stochastic differential delay equation under partial information, Systems Control Lett., 82 (2015), 71-78. 1

[17] X.-M. Xu, Anticipated backward doubly stochastic differential equations, Appl. Math. Comput., 220 (2013), 53-62. 1, 3

[18] J.-M. Yong, Optimality variational principle for controlled forward-backward stochastic differential equations with mixed initial-terminal conditions, SIAM J. Control Optim., 48 (2010), 4119-4156. 1

[19] F. Zhang, Anticipated backward doubly stochastic differential equations, (Chinese) J. Sci. Sin. Math., 43 (2013), 12231236. 1, 3 